Research Article

\title{
A retrospective study of antimicrobial resistance pattern of Pseudomonas aeruginosa isolates from urine samples over last three years (2013-2015)
}

\author{
Neetu Sharma ${ }^{1}$, Anita K. Gupta, Geeta Walia, Rupinder Bakhshi
}

\begin{abstract}
${ }^{1}$ Department of Pharmacology,
${ }^{2}$ Department of Microbiology, Govt. Medical College, Patiala, Punjab, India
\end{abstract}

Received: 29 May 2016

Accepted: 01 July 2016

*Correspondence to:

Dr. Neetu Sharma,

Email: drneetusharma1975@ gmail.com

Copyright: (C) the author(s), publisher and licensee Medip Academy. This is an openaccess article distributed under the terms of the Creative Commons Attribution NonCommercial License, which permits unrestricted noncommercial use, distribution, and reproduction in any medium, provided the original work is properly cited.

\begin{abstract}
Background: Problem of antibiotic resistance is compounding day by day because of overuse and misuse of antibiotics. There is no systematic national surveillance of antibiotic resistance and insufficient data is available to quantify the problem in our country. This study aims at studying the changing pattern of antimicrobial resistance of Pseudomonas aeruginosa isolates from patients of urinary tract infections over last three years.

Methods: A retrospective, record based study carried out based on the records of $\mathrm{C} / \mathrm{S}$ (Culture sensitivity) reports of indoor patients, during past three years (2013 - 2015). The types of organisms causing urinary tract infections were noted and the drugs still effective for the Pseudomonas aeruginosa were noted.

Results: Pseudomonas aeruginosa is inherently resistant to many antimicrobial agents. Analysis of the results year wise indicated that the lowest percentage of resistance manifested against imipenem was $14 \%, 24.48 \%$ and $20.99 \%$ for the years 2013-2015. Resistance for fluoroquinolones decreased over the three consective years from $84.67 \%$ (2013) to $78.27 \%$ (2015). Over the successive years, the resistance to ceftriaxone tends to increase from $80 \%$ (2013) to $92.59 \%$ (2015). C/S reports showed on an average $69 \%$ were resistant to aminoglycosides. Multi drug resistant pseudomonas percentage resistant to fluoroquinolones, third generation cephalosporines and aminoglycosides were $78 \%$ (2013), $78.33 \%$ and $80.33 \%$ (2015) over the consective years.

Conclusions: The antimicrobial resistance patterns are constantly evolving and vary from region to region it has become a necessity to do constant antimicrobial sensitivity surveillance. This will help clinicians to provide safe and effective empirical therapies.
\end{abstract}

Keywords: Antidiabetic agents, Prescriptions, Changing pattern

\section{INTRODUCTION}

Urinary tract infection (UTI) is a common nosocomial infection and an important source of morbidity in the community as well. ${ }^{1,2}$ Pseudomonas aeruginosa being a very resistant organism is resistant to high concentration of salts and dyes, weak antiseptics and commonly used antibiotics. ${ }^{3}$ Pseudomonas aeruginosa (P. aeruginosa) is also an opportunistic human pathogen. ${ }^{4}$

Many epidemiological outcome studies have concluded that infections caused by drug-resistant P. aeruginosa are associated with significant increases in morbidity, mortality, need for surgical intervention, increased length of hospital stay, chronicity and overall cost of treating the infection. $^{5-7} \mathrm{P}$. aeruginosa presents a big therapeutic challenge for treatment of various infections and selection of the appropriate antibiotic as empirical therapy is essential to optimize the clinical outcome. ${ }^{8,9}$ The extensive and inappropriate use of antimicrobial agents has invariably resulted in the development of antibiotic resistance which, in recent years, has become a major problem worldwide. ${ }^{10}$ Resistance pattern of microorganisms vary from country to country, state to state, large hospital to small hospital and hospital to community. Hence, there is a need to conduct areaspecific monitoring studies to profile different pathogens responsible for specific infections and their resistance 
patterns, so as to generate data that would help clinicians to choose the correct empirical treatment.

The estimation of local etiology and susceptibility profile could support the most effective empirical treatment. ${ }^{11}$ Multiple antibiotic resistance in bacterial populations is a pervasive and growing clinical problem, which is recognized as a threat to public health. In our country, the problem of antibiotic resistance is compounding because of overuse and misuse of antibiotics. There is no systematic national surveillance of antibiotic resistance and insufficient data is available to quantify the problem. The aim of this study was to evaluate the in vitro resistance pattern to commonly used antimicrobial agents. This study will further help in formulating most optimal empirical treatment regimen for UTI cases, while awaiting culture sensitivity reports with minimal therapeutic failure.

\section{METHODS}

A retrospective, record based study was carried out in department of pharmacology in collaboration with microbiology department of a teaching tertiary care hospital. The study was carried out based on the records of $\mathrm{C} / \mathrm{S}$ (culture sensitivity) reports of indoor patients, during past three years from January to December of years 2013 to 2015 admitted in wards of Rajindra Hospital and Govt. Medical College Patiala, a tertiary care 1100 bedded hospital. Permission was taken from ethical committee and in-charge of microbiology laboratory prior to the study. All the $\mathrm{C} / \mathrm{S}$ reports of urine samples, maintained in the record registers of microbiology laboratory received from various wards during the period 2013 to 2015 were included and analysed. Reports of Isolates from repeat culture of previously recruited patients and isolates identified as commensals or contaminants were excluded from the study. Number of reports/years for three years of urine samples which were already registered was noted. Only reports with positive Pseudomonas aeruginosa c/s report were considered for this study. Positive reports for Pseudomonas aeruginosa out of total no. of urine samples positive for the years 2013, 2014 and 2015 were 196 (947), 151 (1058) and 86 (1102) respectively. Sample size for the present study was 333 for the three years. The data was collected year wise starting from the year 2013. The provisional diagnosis was noted.

Antibiotic sensitivity pattern of Pseudomonas aeruginosa isolates was determined on Muller Hilton agar plates by Kirby-Bauer disc diffusion method. Isolates were declared as sensitive or resistant on the basis of zone of inhibition following the criteria of clinical laboratory standards institute (CLSI). ${ }^{12}$ The antibiotics tested were broad-spectrum penicillin, third generation cephalosporin, quinolones, aminoglycosides, and carbapenems and nitrofurantoin. Type of organisms most common in urine sample were noted and drugs still effective for the particular organism were noted. Whole of the data was collected and compiled in the year wise manner and compared year wise to see the trend in the resistance pattern. An isolate was considered as MDR if found resistant to three or more antimicrobials belonging to different classes/groups of antimicrobials. ${ }^{12} \mathrm{C} / \mathrm{S}$ reports for other common uropathogens will also be further analysed.

\section{Statistical analysis}

Descriptive statistics was used for analysis. Proportions were used to study the resistance pattern of Pseudomonas aeruginosa and variables were expressed as percentages. Licensed SPSS version 20 was used for statistical analysis. All the data was expressed as Tables and bar Figure.

\section{RESULTS}

Over a 3 year period of 2013-2015, a total of 2,464 positive urine isolates including 333 Pseudomonas aeruginosa were analysed. As an average of $14 \%$ of the total isolates was Pseudomonas aeruginosa while E. coli was the most frequent isolate throughout the three years (average of $67.66 \%$ of the total isolates). Analysis of the results year wise indicated that the lowest percentage of resistance manifested against imipenem was 14\%, $24.48 \%$ and $20.99 \%$ for the years 2013-2015 (Table1).

Table 1: Antibiotic resistance patterns of $P$. aeruginosa against different group of antibiotics.

\begin{tabular}{|llll|}
\hline Antimicrobial agents & \multicolumn{3}{c|}{ Resistant } \\
\hline & $\mathbf{2 0 1 3}$ & $\mathbf{2 0 1 4}$ & $\mathbf{2 0 1 5}$ \\
\hline Piperacillin & 43.33 & 58.62 & 79.01 \\
\hline Aminoglycosides & 68.9 & 69.78 & 69.54 \\
\hline Fluoroquinolones & 84.67 & 79.31 & 78.27 \\
\hline Ceftriaxone & 80 & 85.52 & 92.59 \\
\hline Cefoperazone+sulbactam & 53.33 & 65.52 & 51.85 \\
\hline Imipenem & 14 & 24.48 & 20.99 \\
\hline Nitrofurantoin & 73.33 & 70.69 & 83.95 \\
\hline Ceftazidime+tazobactum & 83.33 & 77.59 & 100 \\
\hline Polymyxin B & 100 & 70.69 & 29.63 \\
\hline
\end{tabular}

Resistance for fluoroquinolones decreased over the three consective years from $84.67 \%$ (2013) to $78.27 \%$ (2015). Over the successive years, the resistance to ceftriaxone, tends to increase from $80 \%$ (2013) to $92.59 \%$ (2015). C/S reports showed on an average $69 \%$ were resistant to aminoglycosides (Figure 1).

\section{MDR (multi-drug resistant) P. aeruginosa}

Those strains which are found to be resistant to three or more than three groups of antimicrobials were taken as multi drug resistant strains. MDR pseudomonas percentage resistant to fluoroquinolones, third generation cephalosporines and aminoglycosides were $78 \%$ (2013), $78.33 \%$ and $80.33 \%$ (2015) over the consective years. 


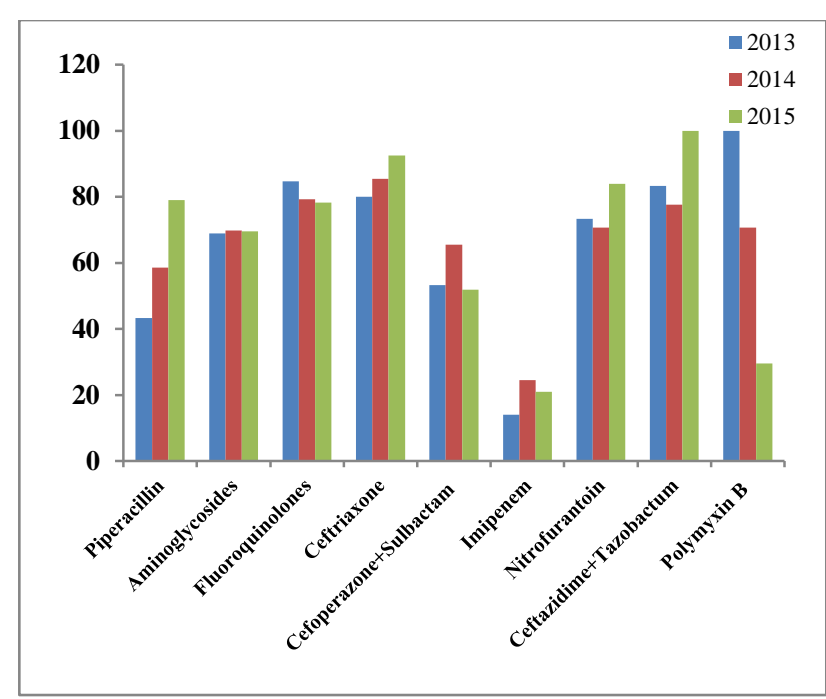

Figure1: Antibiotic resistance trends of Pseudomonas aeruginosa isolates in urine samples over last three years from 2013 to 2015.

\section{DISCUSSION}

This study shows the distribution of microbial species and antibiotic resistance patterns of $\mathrm{P}$. aeruginosa isolated from North Indian patients with UTI. Rajindra hospital is an 1100-bedded hospital located in Patiala, Punjab; it is one of the busiest hospitals in the state. Antibiotic resistance is a major clinical problem in treating infections caused by these microorganisms. The resistance to the antimicrobials has increased over the years. Resistance rates vary from one region to another. ${ }^{13,14}$ There are very few studies done in Punjab for keeping a check on the changing trend in antimicrobial resistance.

Gram-negative organisms are the most common organisms causing UTIs. In this study, an average of $14 \%$ of the total isolates was Pseudomonas aeruginosa while E. coli was the most frequent isolate throughout the three years (average of $67.66 \%$ of the total isolates). The spectrum of uropathogens isolated from urine samples in this study is very similar to the studies done in different regions of India. ${ }^{15-17}$

In our study, P. aeruginosa isolates found to be resistant to aminoglycosides between 69\% (2013) and 70\% (2015). Such high level of resistance of $68 \%$ and $55 \%$ and $70 \%$ was documented from studies done in Gujarat (India), Iran and European studies respectively. ${ }^{18-20}$ Although fluoroquinolones are among the most effective drugs in treating UTI, diverse studies have revealed increasing resistance to fluoroquinolones around $70 \% .{ }^{18}$ Similarly in our study resistance to fluoroquinolones is between $84 \%$ (2013) and 78\% (2015). This may be due to rampant use of fluoroquinolones as first line empirical therapy in UTI cases.
Piperacillin and aminoglycosides are consistently showing high resistance percentage over the successive three years. Third generation cephalosporines like ceftriaxone is showing very obvious increasing trend of resistance over the three years ranging from $80 \%$ (2013) to $92 \%$ (2015). In a study done in Gujarat ceftriaxone resistance is found to be $75 \%$ which is quite similar to our study. ${ }^{18}$ This may be due to the increasing clinical use of third generation cephalosporins following the resistant strains to fluoroquinolones.

Lowest resistance is seen to imipenem in our study between $14 \%$ (2013) and 20\% (2015). This is quite similar to the study done in Merrut, Uttar Pradesh. ${ }^{21}$ High level resistance to nitrofurantoin is seen in our study which is very similar to other studies. ${ }^{19,21}$

In the present study another finding which raises an alarm is about MDR, urinary isolates of P. aeruginosa show $78 \%(2013), 78.33 \%$ and $80.33 \%$ (2015) resistance to fluoroquinolones, third-generation cephalosporins and aminoglycosides collectively. The rising trend of MDR is seen over the successive years.

In a study of antibiotic susceptibility done in Merrut, Uttar Pradesh fluoroquinolone resistance is $15 \% .^{21}$ These results are quite different from our study thereby stressing upon the regional differences and importance of surveillance of antimicrobial resistance and suggestion of empirical therapy accordingly.

In this retrospective study, there is no consideration of patient's demographic data like age, gender etc., and clinical symptoms, complicated versus uncomplicated UTI, which are surely the limitations of this study. On the other hand the large sample size of UTI patients and comparison of three years data are strengths of the study.

\section{CONCLUSION}

Easy over the counter availability of antimicrobial agents and ability of P. aeruginosa to resist antimicrobial agents by their inherent or acquired antimicrobial mechanisms has led to high level of drug resistance. Our study showed that $80 \% \mathrm{P}$. aeruginosa of isolates from urine samples were MDR. Because of this a bacterium resistant to one antibiotic is often much more likely to be resistant to second choice of antibiotics, thereby increasing the chances of failure of therapy in UTI. The antimicrobial resistant patterns are constantly evolving and vary from region to region it is a necessity for constant antimicrobial sensitivity surveillance. This will help clinicians to provide safe and effective empirical therapies with minimal therapeutic failures.

\section{Funding: No funding sources}

Conflict of interest: None declared

Ethical approval: The study was approved by the Institutional Ethics Committee 


\section{REFERENCES}

1. Sharma S. Current understanding of pathogenic mechanisms in UTIs. Ann Natl Acad Med Sci. 1997;33:31.

2. Acharya A, Gautam R, Subedee L. Uropathogens and their antimicrobial susceptibility pattern in Bharatpur, Nepal. Nepal Med Coll J. 2011;13:30-3.

3. Todar, Kenneth: Todar, s Online Textbook of Bacteriology. The Good, the Bad, and the Deadly. SCIENCE Magazine; 2004:304:1421.

4. Vianelli N, Giannini MB, Quartic C. Resolution of a Pseudomonas aeruginosa outbreak in a haematology unit with the use of disposable sterile water filter. Haematol J. 2006;91(7):983-5.

5. Aloush V, Navon-Venezia S, Seigman-Igra Y, Cabili S, Carmeli Y. Multidrug-resistant Pseudomonas aeruginosa: risk factors and clinical impact. Antimicrob. Agents Chemother. 2006;50:43-8.

6. Carmeli Y, Troillet N, Karchmer AW, Samore MH. Health and economic outcomes of antibiotic resistant Pseudomonas aeruginosa. Arch Intern Med. 1999;159:1127-32.

7. Gasink LB, Fishman NO, Weiner MG, Nachamkin I, Bilker WB, Lautenbach E. Fluoroquinolone-resistant Pseudomonas aeruginosa: assessment of risk factors and clinical impact. Am J Med. 2006;119:526e19$526 \mathrm{e} 25$.

8. Bisbe J, Gatell JM, Puig J. Pseudomonas aeruginosa bacteremia: univariate and multivariate analyses of factors influencing the prognosis in 133 episodes. Rev Infect Dis. 1988;10:629-35.

9. Micek ST, Lloyd AE, Ritchie DJ, Reichley RM, Fraser VJ, Kollef MH. Pseudomonas aeruginosa bloodstream infection: importance of appropriate initial antimicrobial treatment. Antimicrob Agents Chemother. 2005;49:1306-11.

10. Goldstein FW. Antibiotic susceptibility of bacterial strains isolated from patients with communityacquired urinary tract infections in France. Multicentre Study Group. Eur J Clin Microbiol Infect Dis. 2000;19:112-7.

11. Farajnia S, Alikhani MY, Ghotaslou R, Naghili B, Nakhlband A. Causative agents and antimicrobial susceptibilities of urinary tract infections in the northwest of Iran. Int J Infect Dis. 2009;13:140-4.

12. Niranjan V, Malini A. Antimicrobial resistance pattern in Escherichia coli causing urinary tract infection among inpatients. Indian J Med Res. 2015;139:945-48.
13. Farrell DJ, Morrissey I, De Rubeis D, Robbins M, Felmingham D. A UK multi centre study of the antimicrobial susceptibility of bacterial pathogens causing urinary tract infection. $\mathrm{J}$ Infect. 2003;46:94-100.

14. Mathai D, Jones RN, Pfaller MA. Epidemiology and frequency of resistance among pathogens causing urinary tract infections in 1,510 hospitalized patients: a report from the SENTRY antimicrobial surveillance program (North America). Diagn Microbiol Infect Dis. 2001;40:129-36.

15. Prakash D, Saxena RS. Distribution and antimicrobial susceptibility pattern of bacterial pathogens causing urinary tract infection in urban community of Meerut City, India. Microbiol. 2014:749629.

16. Mukherjee M, Basu S, Mukherjee SK, Majumder M. Multi drug resistance and extended spectrum betalactamase productionin uropathogenic E. Coli which were isolated from hospitalized patients in Kolkata, India. J Clin Diagn Res. 2014;7:449-53.

17. Akram M, Shahid M, Khan AU. Etiology and antibiotic resistance patterns of community-acquired urinary tract infections in JNMC Hospital Aligarh, India. Ann Clin Microbiol Antimicrob. 2007;6:4.

18. Javiya VA, Ghatak SB, Patel KR, Patel JA. Antibiotic susceptibility patterns of Pseudomonas aeruginosa at a tertiary care hospital in Gujarat, India. Indian J Pharmacol. 2008;40(5):230-4.

19. Amin M, Mehdinejad M, Pourdangchi Z. Study of bacteria isolated from urinary tract infections and determination of their susceptibility to antibiotics. Jundishapur Journal of Microbiology. 2009;2(3):11823.

20. Bouza E, San Juan R, Muñoz P, Voss A, Kluytmans J. Co-operative group of the European study group on nosocomial infections: a European perspective on nosocomial urinary tract infections I. Report on the microbiology workload, etiology and antimicrobial susceptibility (ESGNI-003 study). European Study Group on Nosocomial Infections. Clin Microbiol Infect. 2001;7(10):523-31.

21. Prakash D, Saxena RS. Distribution and antimicrobial susceptibility pattern of bacterial pathogens causing urinary tract infection in urban community of Meerut City, India. ISRN Microbiology. 2014;1:1-13.

Cite this article as: Sharma N, Gupta AK, Walia G, Bakhshi R. A retrospective study of antimicrobial resistance pattern of Pseudomonas aeruginosa isolates from urine samples over last three years (2013-2015). Int J Basic Clin Pharmacol 2016;5:1551-4. 